\title{
Symmetric Argumentation Frameworks
}

\author{
Sylvie Coste-Marquis, Caroline Devred, and Pierre Marquis \\ CRIL-CNRS/Université d'Artois * \\ rue de l'Université - S.P. 16 \\ F-62307 Lens Cedex - France \\ \{coste, devred, marquis\} @cril.univ-artois.fr
}

\begin{abstract}
This paper is centered on the family of Dung's finite argumentation frameworks when the attacks relation is symmetric (and nonempty and irreflexive). We show that while this family does not contain any well-founded framework, every element of it is both coherent and relatively grounded. Then we focus on the acceptability problems for the various semantics introduced by Dung, yet generalized to sets of arguments. We show that only two distinct forms of acceptability are possible when the considered frameworks are symmetric. Those forms of acceptability are quite simple, but tractable; this contrasts with the general case for which all the forms of acceptability are intractable (except for the ones based on grounded or naive extensions).
\end{abstract}

\section{Introduction}

Modelling argumentation is known as a major issue of many AI problems, including defeasible reasoning and some forms of dialogue between agents (see e.g., [1-5]). In a nutshell, argumentative reasoning is concerned with the interaction of arguments. A key notion for any theory of argumentation is the acceptability one: intuitively, an argument is considered acceptable if it can be argued successfully against attacking arguments. Formally, the acceptability of an argument (resp. a set of arguments taken as a whole) is characterized by the membership (resp. the containment) of it to some selected sets of arguments, referred to as extensions.

Several theories of argumentation have been proposed so far (see among others [6-10]). In Elvang-Gøransson et al.'s theory (refined and extended by several authors, including [7,11-19]), one considers in the beginning a set of assumptions and some background knowledge; then an argument is a pair consisting of a statement (the conclusion of the argument) and a (often minimal) subset of assumptions (the support of the conclusion) which is consistent with the background knowledge and such that the conclusion is a logical consequence of it and the background knowledge. Several forms of interaction between arguments have been investigated, including among others the rebuttal relation (an argument rebuts a second one when the conclusion of the former is equivalent to the negation of the conclusion of the latter). In Dung's approach ${ }^{1}$ [6], no assumption is made about the nature of an argument (it can be a statement supported

\footnotetext{
* The authors have been partly supported by the the Région Nord/Pas-de-Calais through the IRCICA Consortium and by the European Community FEDER Program.

${ }^{1}$ Also refined and extended by several authors, including [20-24].
} 
by some assumptions like in the theory introduced by Elvang-Gøransson et al. but this is not mandatory). What really matters is the way arguments interact w.r.t. the attacks relation. In contrast to Elvang-Gøransson et al.'s theory, Dung's theory of argumentation is not concerned with the generation of arguments; arguments and the way they interact are considered as initial data of any argumentation framework. Several notions of extensions have been defined by Dung, reflecting several reasons according to which arguments can be taken together. A major feature of Dung's theory is that it encompasses many approaches to nonmonotonic reasoning and logic programming as special cases.

In this paper, we focus on the family of finite argumentation frameworks obtained by requiring the attacks relation to be symmetric; we also assume that the attacks relation is not empty (which is not so strong an assumption since the argumentation frameworks which violate it are trivial ones: no interactions between arguments exist) and that it is irreflexive; the latter assumption is also sensible since an argument which attacks itself is in some sense paradoxical and the problem of reasoning with paradoxical statements is hard by itself but mainly independent from the argumentation issue. Thus, paradoxical statements are typically not viewed as arguments (for instance, it cannot be the case that the support of a conclusion contradicts the conclusion in Elvang-Gøransson et al.'s approach). The symmetry requirement is also not so strong; for instance, the rebuttal relation in Elvang-Gøransson et al.'s theory is clearly symmetric.

Our contribution is twofold. We show that while no symmetric argumentation framework is also well-founded, every symmetric argumentation framework is both coherent and relatively grounded. Then we focus on the acceptability problems for the various semantics introduced by Dung, yet generalized to sets of arguments. We show that only two distinct forms of acceptability are possible when considering symmetric frameworks. Finally, we show that those forms of acceptability are quite simple, but tractable for symmetric frameworks, while they are intractable in the general case (except for the ones based on grounded or naive extensions).

The rest of this paper is organized as follows. In Section2, we recall the main definitions and results pertaining to Dung's theory of argumentation. In Section 3, we focus on symmetric argumentation frameworks and present our contribution. Finally, Section 4 concludes the paper.

\section{Dung's Theory of Argumentation}

Let us present some basic definitions at work in Dung's theory of argumentation [6]. We restrict them to finite argumentation frameworks.

Definition 1 (finite argumentation frameworks).

$A$ finite argumentation framework is a pair $A F=\langle A, R\rangle$ where $A$ is a finite set of so-called arguments and $R$ is a binary relation over $A$ (a subset of $A \times A$ ), the attacks relation.

Clearly enough, the set of finite argumentation frameworks is a proper subset of the set of Dung's finitary argumentation frameworks, where every argument must be attacked by finitely many arguments. 
The definition above clearly shows that a finite argumentation framework is nothing but a finite digraph.

Example 1. Let $A F=\langle A, R\rangle$ be a finite argumentation framework with $A=\{a, b, c, d, e\}$ and $R=\{(e, c),(c, e),(b, c),(c, b),(b, d),(b, d),(c, d),(d, c)\}$.

$A F$ is depicted on Figure 1. One can observe that $R$ is a symmetric relation; clearly, this is not always the case for Dung's frameworks but this choice is motivated by the desire to take advantage of $A F$ as a running example throughout the paper.

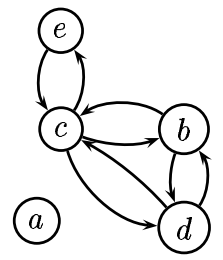

Fig. 1. Digraph for $A F$.

A first important notion is the notion of acceptability: an argument $a$ is acceptable w.r.t. a set of arguments whenever it is defended by the set, i.e., every argument which attacks $a$ is attacked by an element of the set.

Definition 2 (acceptability w.r.t. a set).

Let $A F=\langle A, R\rangle$ be a finite argumentation framework. An argument $a \in A$ is acceptable w.r.t. a subset $S$ of $A$ if and only if for every $b \in A$ s.t. $(b, a) \in R$, there exists $c \in S$ s.t. $(c, b) \in R$. A set of arguments is acceptable w.r.t. $S$ when each of its elements is acceptable w.r.t. $S$.

In the graph theory literature, a set of vertices which is acceptable w.r.t. itself is said to be semidominant.

A second important notion is the notion of absence of conflicts. Intuitively, two arguments should not be considered together whenever one of them attacks the other one.

Definition 3 (conflict-free sets).

Let $A F=\langle A, R\rangle$ be a finite argumentation framework. A subset $S$ of $A$ is conflict-free if and only if for every $a, b \in S$, we have $(a, b) \notin R$.

The conflict-free subsets of $A$ which are maximal w.r.t. $\subseteq$ are called the naive extensions of $A F$ in [3]. In the graph theory literature, such conflict-free sets are also called independent sets.

Requiring the absence of conflicts and the form of autonomy captured by selfacceptability leads to the notion of admissible set.

Definition 4 (admissible sets).

Let $A F=\langle A, R\rangle$ be a finite argumentation framework. A subset $S$ of $A$ is admissible if and only if $S$ is conflict-free and acceptable w.r.t. $S$. 
In the graph theory literature, a set of vertices which is both independent and semidominant is called a semikernel.

Example 2 (Example 1 (cont'ed)). $\{e, d\},\{e, b\},\{c\}$ are admissible sets given $A F$.

The significance of the concept of admissible sets is reflected by the fact that every extension of an argumentation framework under the standard semantics introduced by Dung (preferred, stable, complete and grounded extensions) is an admissible set, satisfying some form of optimality:

Definition 5 (extensions).

Let $A F=\langle A, R\rangle$ be a finite argumentation framework.

- A subset $S$ of $A$ is a preferred extension of $A F$ if and only if it is maximal w.r.t. $\subseteq$ among the set of admissible sets for $A F$.

- A subset $S$ of $A$ is a stable extension of $A F$ if and only if it is admissible and for every argument a from $A \backslash S$, there exists $b \in S$ s.t. $(b, a) \in R$.

- A subset $S$ of $A$ is a complete extension of $A F$ if and only if it is admissible and it coincides with the set of arguments acceptable w.r.t. itself.

- A subset $S$ of $A$ is the grounded extension of $A F$ if and only if it is the least element w.r.t. $\subseteq$ among the complete extensions of $A F$.

Example 3 (Example 1 (cont'ed)). Let $E_{1}=\{a\}, E_{2}=\{a, e, b\}, E_{3}=\{a, c\}$ and $E_{4}=\{a, d, e\} . E_{1}$ is the grounded extension of $A F . E_{2}, E_{3}$ and $E_{4}$ are the preferred extensions of $A F$ and the stable extensions of $A F . E_{1}, E_{2}, E_{3}$ and $E_{4}$ are the complete extensions of $A F$.

In the graph theory literature, sets $S$ of vertices s.t. every vertex outside $S$ is in the direct image of at least one element of $S$ are also called dominating sets. Sets of vertices that are both independent and dominating are referred to as the kernels of the graph $A F$. The sets of vertices which are the maximal semikernels of the graph $A F$ are the preferred extensions of $A F$.

Formally, complete extensions of $A F$ can be characterized as the fixed points of its characteristic function $\mathcal{F}_{A F}$, and among them, the grounded extension of $A F$ is the least element [6]:

Definition 6 (characteristic functions).

The characteristic function, denoted $\mathcal{F}_{A F}$, of an argumentation framework $A F=$

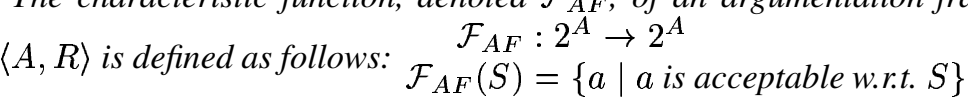

Finally, several notions of acceptability of an argument (or more generally a set of arguments) can be defined by requiring the membership to one (credulous acceptability) or every extension (skeptical acceptability) of a specific kind. Obviously enough, credulous acceptability and skeptical acceptability w.r.t. the grounded extension coincide, since the grounded extension of an argumentation framework is unique.

Among other things, Dung has shown that every argumentation framework $A F$ has at least one preferred extension, while it may have zero, one or many stable extensions. 
The purest argumentation frameworks $A F$ in Dung's theory are those for which all the notions of acceptability coincide. This means that $A F$ has a unique complete extension (the grounded one), which is also stable and preferred.

Definition 7. An argumentation framework $A F=\langle A, R\rangle$ is well-founded if and only if there does not exist an infinite sequence $a_{0}, a_{1} \ldots a_{n} \ldots$ of arguments from $A$, such that for each $i,\left(a_{i+1}, a_{i}\right) \in R$.

Proposition 1. Every well-founded argumentation framework has exactly one complete extension which is grounded, preferred and stable.

Dung has provided a sufficient condition for an argumentation framework $A F$ to satisfy this requirement, the well-foundation of $A F$ :

Proposition 2. Let $A F=\langle A, R\rangle$ be a finite argumentation framework. $A F$ is wellfounded if there is no cycle in the digraph $\langle A, R\rangle$.

Dung has also shown that every stable extension is preferred and every preferred extension is complete; however, none of the converse inclusions holds. When all the preferred extensions of an argumentation framework are stable ones, the framework is said to be coherent:

Definition 8 (coherent argumentation frameworks).

Let $A F=\langle A, R\rangle$ be a finite argumentation framework. $A F$ is coherent if and only if every preferred extension of $A F$ is also stable.

Example 4 (Example 1 (cont'ed)). Every preferred extension of $A F$ is a stable extension as well. Hence $A F$ is coherent.

This is particularly interesting since for any coherent $A F$, the notion of credulous (resp. skeptical) acceptability w.r.t. the preferred arguments coincides with the notion of credulous (resp. skeptical) acceptability w.r.t. the stable arguments.

Since the grounded extension of $A F$ is the least complete extension of it, it is included in every preferred extension of $A F$ (hence in every stable extension of $A F$ ). This shows that the notion of acceptability w.r.t. the grounded extension is always at least as demanding as any form of credulous or skeptical acceptability w.r.t. the preferred extensions or the stable ones (except for credulous acceptability w.r.t. the stable extensions when no such extensions exist since no argument can be accepted in that case for such semantics - note that such an exception cannot be the case when $A F$ is coherent).

Nevertheless, the grounded extension of $A F$ is not always equal to the intersection of all its preferred extensions. Interesting argumentation frameworks are those for which this condition is satisfied:

Definition 9 (relatively grounded argumentation frameworks).

Let $A F=\langle A, R\rangle$ be a finite argumentation framework. $A F$ is relatively grounded if and only if its grounded extension is equal to the intersection of all its preferred extensions.

Example 5 (Example 1 (cont'ed)). $E_{2} \cap E_{3} \cap E_{4}=E_{1}$. Hence $A F$ is relatively grounded.

In this case, the notion of skeptical acceptability w.r.t. the preferred extensions coincides with the notion of acceptability w.r.t. the grounded extension. 


\section{Symmetric Argumentation Frameworks}

\subsection{Definitions and properties}

Let us now make precise the argumentation frameworks we are interested in.

Definition 10 (symmetric argumentation frameworks).

A symmetric argumentation framework is a finite argumentation framework $A F=$ $\langle A, R\rangle$ where $R$ is assumed symmetric, nonempty and irreflexive.

Example 6 (Example 1 (cont'ed)). AF is a symmetric argumentation framework.

First of all, it is easy to show that no symmetric argumentation framework is among the purest ones:

Proposition 3. No symmetric argumentation framework is well-founded.

Proof. Since $R$ is nonempty and symmetric, a cycle can always be found in $A F$.

Nevertheless, this does not prevent symmetric argumentation frameworks from exhibiting interesting properties. An easy result is:

Proposition 4. Let $A F=\langle A, R\rangle$ be a symmetric argumentation framework. $S \subseteq A$ is admissible if and only if $S$ is conflict-free.

Proof. Since $R$ is symmetric, every argument $a$ of $A$ defends itself against all the arguments which attack it, so every $a \in A$ is acceptable w.r.t $a$. Hence, for all $S \subseteq A$, every $a \in A$ is acceptable w.r.t. $S \cup\{a\}$. Hence, for all $S \subseteq A$, every $a \in S$ is acceptable w.r.t. $S$. Hence, $S$ is admissible if $S$ is conflict-free.

Thus, the preferred extensions of a symmetric $A F=\langle A, R\rangle$ are the maximal subsets of $A$ w.r.t. $\subseteq$ among those which are conflict-free, i.e. the naive extensions of $A F$ [3]. In particular, every conflict-free subset of $A$ is included in a preferred extension of $A F$.

Another consequence is that:

Proposition 5. Every symmetric argumentation framework is coherent.

Proof. Every preferred extension $E \subseteq A$ is a naive extension. Hence, each argument not in $E$ is in conflict with $E$. Since R is symmetric, each argument not in $E$ is attacked by $E$. Hence, $E$ is a stable extension.

Since every symmetric argumentation framework has a preferred extension, every symmetric argumentation framework has a stable extension, which is necessarily nonempty. Actually, this is an easy consequence of a more general result from graph theory stating that symmetric graphs are kernel perfect. This means that every induced subgraph of a symmetric graph has a kernel.

Proposition 6. Let $A F=\langle A, R\rangle$ be a symmetric argumentation framework. Every $a \in A$ belongs to at least one preferred (or equivalently, stable or naive) extension of $A F$. 
Proof. Immediate, since $R$ is irreflexive and symmetric.

Example 7 (Example 1 (cont'ed)). $E_{2} \cup E_{3} \cup E_{4}=A$. Hence every argument of $A$ belongs to a preferred extension of $A F$.

As to the grounded extension, we can prove that:

Proposition 7. Let $A F=\langle A, R\rangle$ be a symmetric argumentation framework. The grounded extension of $A F$ is given by $\{a \in A \mid \nexists b \in A,(b, a) \in R\}$.

Proof. According to Definition $6, \mathcal{F}_{A F}(\emptyset)$ is the set of arguments of $A F$ which are not attacked. There are two cases:

1. Either every argument of $A$ is attacked. Then $\mathcal{F}_{A F}(\emptyset)=\emptyset$ is the least complete extension of $A F$ (w.r.t. $\subseteq$ ). Hence $\emptyset$ is the grounded extension of $A F$.

2. Or some arguments of $A$ are not attacked. Let $S^{\prime}=\mathcal{F}_{A F}(\emptyset)$ be the set of such arguments. Since $R$ is symmetric, if an argument is not attacked, then it does not attack any argument. Hence, there is no $a \in A \backslash S^{\prime}$ s.t. $a$ is acceptable w.r.t. $S^{\prime}$. Hence $\mathcal{F}_{A F}^{2}(\emptyset)=\mathcal{F}_{A F}\left(S^{\prime}\right)=S^{\prime}$. So, $S^{\prime}$ is the least complete extension of $A F$ (w.r.t. $\subseteq$ ). Hence $S^{\prime}$ is the grounded extension of $A F$.

Subsequently, the grounded extension of $A F$ can be computed in time linear in $|A F|$ in the worst case.

We have also shown that:

Proposition 8. Let $A F=\langle A, R\rangle$ be a symmetric argumentation framework. $a \in A$ belongs to every preferred (or equivalently, stable or naive) extension of $A F$ if and only if there is no $b \in A$ s.t. $(b, a) \in R$.

Proof.

$\Leftarrow$ Immediate from Proposition 7 and the fact that the grounded extension is included into every preferred extension.

$\Rightarrow$ Let $b \in A$ such that $(b, a) \in R$. According to Proposition 6, there is a preferred extension $E$ such that $b \in E$. But $a$ belongs to $E$. Thus $E$ is not conflict-free. So, $b$ does not exist.

A direct corollary of this proposition is the following one:

Proposition 9. Every symmetric argumentation framework is relatively grounded.

Proof. Immediate from Propositions 7 and 8.

Example 8 (Example 1 (cont'ed)). $a$ is not attacked. $a$ belongs to every preferred extension of $A F$ and it is the unique argument of the grounded extension $E_{1}$ of $A F$. 
As a consequence, there are at most two distinct forms of acceptability for symmetric argumentation frameworks: all the forms of skeptical acceptability coincide with the notion of acceptability w.r.t. the grounded extension; credulous acceptability w.r.t. preferred extensions and credulous acceptability w.r.t. stable extensions coincide with credoulous acceptability w.r.t. naive extensions. Nevertheless, according to Proposition 6 credulous acceptability for single arguments is not so interesting since it trivializes for symmetric argumentation frameworks.

Accordingly, one has to consider more general acceptability problems if one wants to get more than one semantics, which is expected here; indeed, skeptical acceptability is rather poor since it characterizes as acceptable only those arguments of $A$ which are not attacked.

\subsection{Acceptability problems and complexity issues}

This is why we turn to acceptability problems for sets of arguments, i.e., the question is now to determine whether or not it is reasonable to accept some arguments together:

\section{Definition 11 (acceptability problems).}

ACCEPTABILITY $_{I, E}$ is the following decision problem (also viewed as the language of its positive instances in the usual way):

- Input: A finite argumentation framework $A F=\langle A, R\rangle$ and a set of arguments $S \subseteq A$.

- Question: Is $S$ included into:

$I=\forall$ : every $E$ extension of $A F$ ?

$I=\exists:$ at least one $E$ extension of $A F$ ?

where $E$ is either $N$ (naive), $P$ (preferred), $S$ (stable), $C$ (complete) or $G$ (grounded).

For instance, ACCEPTABILITY $\forall, S$ denotes the skeptical acceptability problem under the stable semantics. We also use the notation ACCEPTABILITY,,$G$ to denote the acceptability problem under the grounded semantics (obviously enough, ACCEPTABILITY., $G$ $=$ ACCEPTABILITY $_{\forall, G}=$ ACCEPTABILITY $_{\exists, G}$ since an argumentation framework always has a unique grounded extension).

We can easily complete previous complexity results for skeptical acceptability of single arguments $[25,26]$ :

Proposition 10. The following complexity results hold: ${ }^{2}$

- ACCEPTABILITY $\forall, P$ is $\Pi_{2}^{p}$-complete.

- ACCEPTABILITY $\forall, S$ is coNP-complete.

- ACCEPTABILITY $_{\forall, C}=$ ACCEPTABility $_{., G}$ is in $\mathrm{P}$.

- ACCEPTABILITY $\forall, N$ is in $\mathrm{P}$.

\footnotetext{
${ }^{2}$ We assume the reader acquainted with basic notions of complexity theory; see e.g., [27] otherwise.
} 
Proof. Clearly enough, considering sets of arguments has no impact w.r.t. skeptical acceptability whatever the underlying semantics: a set $S$ of arguments is skeptically acceptable if and only if $S$ is a subset of all the extensions under consideration if and only if every element of $S$ is skeptically acceptable. Hence the complexity of skeptical acceptability for sets of arguments coincides with the corresponding complexity of skeptical acceptability for single arguments, as identified by Dunne and Bench-Capon (when the set of arguments is finite and the attacks relation is not empty) [26]. Now, since the grounded extension of an argumentation framework $A F$ is the intersection of all its complete extensions, it also comes that the two languages ACCEPTABILITY ${ }_{\forall, C}$ and ACCEPTABILITY., $G$ coincide. Finally, a set of arguments $S$ is included into every naive extension of $A F=\langle A, R\rangle$ if and only if $S$ is conflict-free and for every argument $a \in A \backslash S$ and every argument $b \in S$ if $(a, b) \in R$ then $(a, a) \in R$. This can be tested in time polynomial in $|A F|+|S|$.

The picture is not the same when credulous acceptability is considered since it can be the case that both arguments $a$ and $b$ are credulously acceptable (this is always the case in presence of symmetric argumentation frameworks) but that the set $\{a, b\}$ does not belong to any of the selected extensions.

Example 9 (Example 1 (cont'ed)). $c \in E_{3}$ and $d \in E_{4}$. Hence each of $c$ and $d$ is credulously acceptable. However, it is not cautious to believe in the set of arguments $\{c, d\}$ because this set is not conflict-free.

Nevertheless, considering sets of arguments instead of arguments alone does not lead to a complexity shift:

Proposition 11. The following complexity results hold:

- ACCEPTABILITY $_{\exists, P}=$ ACCEPTABILITY $_{\exists, C}$ is NP-complete.

- ACCEPTABILITY $\exists, S$ is NP-complete.

- ACCEPTABILITY $\exists, N$ is in $\mathrm{P}$.

Proof. The equality ACCEPTABILITY ${ }_{\exists, P}=$ ACCEPTABILITY $_{\exists, C}$ comes easily from the fact that the preferred extensions of an argumentation framework $A F$ are exactly the complete extensions of $A F$ which are maximal w.r.t. $\subseteq$ (this is a straightforward consequence of the fact that every preferred extension of $A F$ is a complete extension of $A F$ and that every admissible set of arguments of $A F$ (including its complete extensions) is included in a preferred extension of $A F$ (Theorem 2 from [6])). Then the membership results come from the following nondeterministic algorithms running in time polynomial in the input size: guess $S^{\prime} \subseteq A$ then check that $S^{\prime}$ is a complete (resp. stable) extension of $A F$ and that $S \subseteq S^{\prime}$. It is easy to show that the check step can be done in (deterministic) polynomial time. The hardness results are direct consequences of the fact that their restrictions to the case $S$ contains a single argument are already NP-hard $[25,26]$. Finally checking whether a set $S$ of argument belongs to a naive extension is equivalent to checking whether $S$ is conflict-free, which can be done easily in polynomial time. 
One can observe that the notion of complete extension does not lead to semantics which differ from semantics obtained when some other extensions are considered (thus, skeptical acceptability w.r.t. complete extensions coincides with acceptability w.r.t. the grounded extension while credulous acceptability w.r.t. complete extensions coincides with credulous acceptability w.r.t. preferred extensions); this explains why in Dung's work the notion of complete extension is viewed more as a link between preferred extensions and the grounded one than as a semantics per se.

Now, considering symmetric frameworks leads complexity to decrease in a significant way:

Proposition 12. Let us consider the restriction of ACCEPTABILITY ${ }_{I, E}$ when $A F$ is symmetric. Under this requirement, one can prove that:

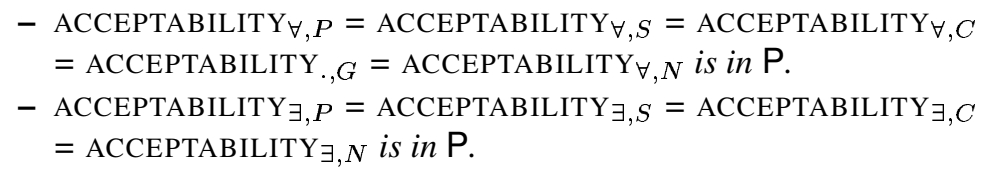

Proof. The first point is a direct consequence of Propositions 7 and 8 . The equalities at the second point come from Propositions 4 and 5 and from the facts that the preferred extensions of an argumentation framework $A F$ are exactly the complete extensions of $A F$ which are maximal w.r.t. $\subseteq$ and that every admissible set of arguments of $A F$ (including its complete extensions) is included in a preferred extension of $A F$ (see the proof of Proposition 11). Tractability comes from Proposition 4: $S \subseteq A$ is included in a preferred extension of $A F$ - or equivalently, included in a stable extension or included in a complete extension or included in a naive extension - if and only if $S$ is conflictfree. Note that while credulous acceptability can be decided easily, the notion does not trivialize when $S$ is not a singleton (which means that the set of positive instances is not always the set of all instances of the problem).

To sum up, the various semantics in Dung's theory applied to symmetric frameworks lead to consider a set of arguments as acceptable when (1) every element of it is not attacked (the skeptical acceptability) or (2) it is conflict-free (the credulous acceptability). In both cases, acceptability can be decided in an efficient way.

\section{Conclusion}

We have studied the properties offered by symmetric argumentation frameworks, under the (quite realistic) assumptions that the set of arguments is finite and the attacks relation is nonempty and irreflexive. Such frameworks are shown coherent and relatively grounded. This ensures that the various notions of acceptability proposed so far reduce at most to two. Extending them to sets of arguments, one obtains two notions of acceptability which are rather simple in essence but tractable; we have shown that this contrasts with the general case for which all the generalized forms of acceptability are intractable (under the usual assumptions of complexity theory), except for the ones based on grounded or naive extensions. 
This work calls for several perspectives. One of them consists in investigating other preference criteria as a basis for additional semantics for argumentation frameworks. Indeed, refining preferred extensions can prove valuable whenever skeptical (resp. credulous) acceptability w.r.t. preferred extensions is considered too cautious (resp. too liberal). For instance, one can select the preferred extensions which are maximal w.r.t. cardinality. On can also associate to every preferred set $S$ of arguments of $A F$ the sum (or the maximum) of the numbers of attacks against each element of $S$; on this ground, one can prefer the admissible sets associated to the least numbers if one thinks that a set of arguments which is not attacked is better than a set of arguments which is massively attacked. One can also adhere to the opposite point of view and prefer in a Popperian style sets of arguments which are robust enough to survive to many attacks. A second perspective consists in investigating the acceptability issue from the complexity point of view whenever a limited amount of non symmetric attacks is allowed. Finally, it would be interesting to point out other graph-theoretic properties for argumentation frameworks which would ensure tractable inference under various semantics.

\section{References}

1. Toulmin, S.: The Uses of Argument. Cambridge University Press (1958)

2. Prakken, A., Vreeswijk, G.: Logics for defeasible argumentation. Volume 4 of Handbook of Philosophical Logic, Second edition. Kluwer Academic Publishers (2002) 219-318

3. Bondarenko, A., Dung, P.M., Kowalski, R., Toni, F.: An abstract, argumentation-theoretic approach to default reasoning. Artificial Intelligence 93 (1997) 63-101

4. Parsons, S., Sierra, C., Jennings, N.: Agents that reason and negotiate by arguing. Journal of Logic and Computation 8 (1998) 261-292

5. Parsons, S., Wooldrige, M., Amgoud, L.: Properties and complexity of some formal interagent dialogues. Journal of Logic and Computation 13 (2003) 348-376

6. Dung, P.M.: On the acceptability of arguments and its fundamental role in nonmonotonic reasoning, logic programming and n-person games. Artificial Intelligence 77 (1995) 321358

7. Elvang-Gøransson, M., Fox, J., Krause, P.: Dialectic reasoning with inconsistent information. In: Proceedings of the $9^{t h}$ Conference on Uncertainty in Artificial Intelligence. (1993) 114 121

8. Pollock, J.: How to reason defeasibly. Artificial Intelligence 57 (1992) 1-42

9. Simari, G., Loui, R.: A mathematical treatment of defeasible reasoning and its implementation. Artificial Intelligence 53 (1992) 125-157

10. Vreeswijk, G.: Abstract argumentation systems. Artificial Intelligence 90 (1997) 225-279

11. Elvang-Gøransson, M., Fox, J., Krause, P.: Acceptability of arguments as logical uncertainty. In: Proceedings of the European Conference on Symbolic and Quantitative Approaches to Reasoning and Uncertainty. (1993) 85-90

12. Elvang-Gøransson, M., Hunter, A.: Argumentative logics: Reasoning with classically inconsistent information. Data and Knowledge Engineering 16 (1995) 125-145

13. Besnard, P., Hunter, A.: A logic-based theory of deductive arguments. Artificial Intelligence 128 (2001) 203-235

14. Amgoud, L., Cayrol, C.: On the acceptability of arguments in preference-based argumentation. In: Proceedings of the $14^{\text {th }}$ Conference on Uncertainty in Artificial Intelligence. (1998) $1-7$ 
15. Amgoud, L., Cayrol, C.: Inferring from inconsistency in preference-based argumentation frameworks. Journal of Automated Reasoning 29 (2002) 125-169

16. Amgoud, L., Cayrol, C.: A reasoning model based on the production of acceptable arguments. Annals of Mathematics and Artificial Intelligence 34 (2002) 197-215

17. Cayrol, C.: From non-monotonic syntax-based entailment to preference-based argumentation. In: Proceedings of the $3^{\text {rd }}$ European Conference on Symbolic and Quantitative Approaches to Reasoning and Uncertainty. Volume 946 of Lecture Notes on Artificial Intelligence. (1995)

18. Cayrol, C.: On the relation between argumentation and non-monotonic coherence-based entailment. In: Proceedings of the $14^{t h}$ International Joint Conference on Artificial Intelligence. (1995)

19. Dimopoulos, Y., Nebel, B., Toni, F.: On the computional complexity of assumption-based argumentation for default reasoning. Artificial Intelligence 141 (2002) 57-78

20. Baroni, P., Giacomin, M., G.Guida: Extending abstract argumentation systems theory. Artificial Intelligence 120 (2000) 251-270

21. Baroni, P., Giacomin, M.: Solving semantic problems with odd-length cycles in argumentation. In: Proceedings of the $7^{t h}$ European Conference on Symbolic and Quantitative Approaches to Reasoning with Uncertainty. Volume 2711 of Lecture Notes on Artificial Intelligence. (2003) 440-451

22. Baroni, P., Giacomin, M.: A recursive approach to argumentation: motivation and perspectives. In: Proceedings of the $10^{t h}$ International Workshop on Non-Monotonic Reasoning. (2004) 50-58

23. Cayrol, C., Doutre, S., Lagasquie-Schiex, M.C., Mengin, J.: Minimal defence: a refinement of the preferred semantics for argumentation frameworks. In: Proceedings of the $9^{\text {th }}$ International Workshop on Non-Monotonic Reasoning. (2002) 408-415

24. Cayrol, C., Lagasquie-Schiex, M.C.: Gradual handling of contradiction in argumentation frameworks. In: Proceedings of the $9^{\text {th }}$ International Conference on Information Processing and Management of Uncertainty in Knowledge-based Systems. (2002) 83-90

25. Dimopoulos, Y., Torres, A.: Graph theoretical structures in logic programs and default theories. Theoretical Computer Science 170 (1996) 209-244

26. Dunne, P., Bench-Capon, T.: Coherence in finite argument system. Artificial Intelligence 141 (2002) 187-203

27. Papadimitriou, C.: Computational complexity. Addison-Wesley (1994) 